\title{
A Combinatorial Approach to Metamaterials Discovery
}

By Eric Plum*, Kenji Tanaka, Wei T. Chen, Vassili A. Fedotov, Din P. Tsai and Nikolay I. Zheludev

[*] Dr. E. Plum, Dr. K. Tanaka, Dr. V. A. Fedotov, Prof. N. I. Zheludev

Optoelectronics Research Centre

University of Southampton

Southampton, SO17 1BJ (UK)

E-mail: erp@orc.soton.ac.uk

Dr. K. Tanaka

Sony Corporation

Shinagawa-ku

Tokyo, 141-0001 (Japan)

W. T. Chen, Prof. D. P. Tsai

Department of Physics

National Taiwan University

Taipei, 10617 (Taiwan)

Keywords: metamaterials, combinatorial engineering, Fano-resonance

Some fifteen years ago a paper reporting a combinatorial approach to materials discoveries revolutionized materials research and other disciplines such as chemistry and pharmacology by demonstrating a method for high-throughput parallel synthesis and analysis of novel artificial chemical compounds. ${ }^{[1]}$ Here we report on the first application of the combinatorial approach to discovery of electromagnetic metamaterials and their optimization.

Electromagnetic metamaterials are manmade media with all sorts of unusual functionalities that can be achieved by artificial structuring on a smaller length scale than that of the external stimulus. We apply the combinatorial approach to the optimization of an important class of plasmonic metamaterials supporting Fano resonances ${ }^{[2]}$ that recently became a prime platform for new switching, gain and sensing applications, slow light and polarization control devices (for a review see [3]). These metamaterials are arrays of asymmetric split ring metallic wire resonators or complementary arrays of ring slits in a metal film. Their application potential depends on the presence and characteristics of reflection and transmission peaks associated with a particular mode of electromagnetic excitation that is weakly coupled to free-space (closed or trapped mode). The characteristics of this resonance are very sensitive to the environment and are responsive to its changes which makes them a prime choice for active metamaterial applications. These resonances can be tailored by design: their spectral position, width and depth depend on many parameters, most notably on the type of metal used for their fabrication, size of the ring and characteristics of the split. ${ }^{[4-7]}$ Optimization of resonance characteristics of these metamaterial structures is relatively straightforward in the microwave and terahertz parts of the spectrum using numerical electromagnetic modeling tools. For the optical part of the spectrum when the meta-molecules should be structured on the nanoscale numerical modeling becomes considerably less reliable for two reasons. First, accurate reproduction of the idealized design parameters is not possible due to limitations of the nanofabrication technologies, in particular sharp edges and ideal vertical cuts are not possible to achieve while the surface of metals has roughness on a scale comparable with the smallest features of the design. Second, electromagnetic material properties used in calculations, in particular those of nanostructured metals are not known to the necessary accuracy and are normally replaced by those of bulk media. However, they can be drastically different from 
those of bulk metals due to the higher role of surface electron and defect scattering in granulated and nanostructured metals. The unsatisfactory character of this substitute is well recognized by the metamaterials research community where in some calculations arbitrarily corrected values of bulk dielectric parameters are used for nanostructured metals to achieve a better fit to experimental data.

The combinatorial approached presented below aims to address the difficulty of optimizing photonic metamaterial designs through computer modeling by resorting to a parallel synthesis and consecutive optical characterization of a large number of spatially addressable nanofabricated metamaterial samples (libraries, see Fig. 1a) with quasi-continuous variation of design parameters. We also compare the results of combinatorial optimization with those of numerical modeling.

(a)

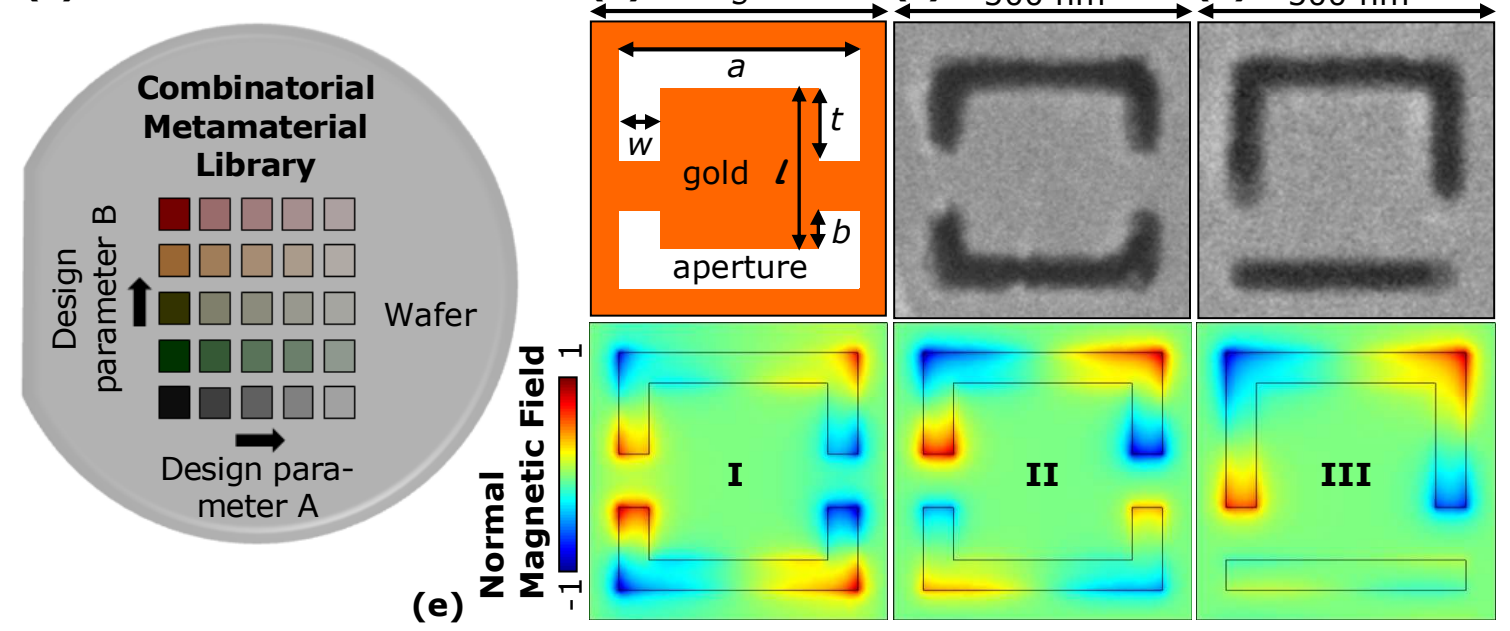

Figure 1. Combinatorial library, meta-molecules and resonant modes. a) Schematic of a combinatorial metamaterial library. b) Schematic of the metamaterial unit cell. c) and d) show scanning electron micrographs of metamaterial unit cells of size $s=500 \mathrm{~nm}$ with small and large gap position asymmetries, $\beta=1 / 7$ and 1. e) Characteristic I symmetric and II-III antisymmetric modes of excitation associated with the absorption resonances for asymmetries $\beta=1 / 7$ and 1 .

We studied metamaterials consisting of arrays of square split-ring aperture meta-molecules in the field of parameters defined by the overall size $s$ of the metamaterial unit cell and the asymmetry of the split (see Fig. 1b). We manufactured two metamaterial libraries which are described in detail in the experimental section. In library A we fixed parameter $b=0$ and introduced a gap size asymmetry $\alpha=t / l$, while in library B a gap position asymmetry $\beta=(t-b) /(t+b)$ was introduced for a fixed gap size of $0.3 l$.

Figure 2a-c illustrates for a fixed meta-molecule size of $s=500 \mathrm{~nm}$ how the metamaterial's transmission, reflection and absorption properties depend on the gap size asymmetry $\alpha=t / l$ when $b=0$. In the symmetric case the metamaterial has broad reflection and transmission maxima around $800 \mathrm{~nm}$ and $1400 \mathrm{~nm}$ respectively, while the absorption spectrum is featureless. Symmetry breaking, $\alpha>0$, leads to the appearance of a Fano resonance near 900 $\mathrm{nm}$ which is associated with a narrow peak in absorption. Furthermore, at slightly shorter wavelengths, a window of transparency opens up, which may be discussed in terms of electromagnetically induced transparency. ${ }^{[8]}$ Larger symmetry breaking leads to broadening of the absorption resonance, which red-shifts due to the increasing size of the top aperture. Figure 3a-c shows how the metamaterial transmission, reflection and absorption depend on the gap position asymmetry $\beta=(t-b) /(t+b)$ for a fixed $90 \mathrm{~nm}$ gap and a unit cell size of 
$s=500 \mathrm{~nm}$. Similarly to the gap size asymmetry discussed above, a gap position asymmetry

$\beta>0$ leads to the appearance of a Fano resonance at $1000 \mathrm{~nm}$ which broadens and red-shifts with increasing asymmetry. Intriguingly, simulations reveal an additional narrow absorption resonance at $830 \mathrm{~nm}$, which should be supported by the symmetric structure and vanishes with increasing asymmetry $\beta$. The fundamentally different nature of these resonances becomes clear when comparing the associated modes of excitation shown in Fig. 1e. The symmetric structure supports a symmetric mode $\mathbf{I}$, where top and bottom apertures interact through in-phase magnetic fields. Symmetry breaking allows the excitation of an antisymmetric mode II, where the apertures of each split ring interact through anti-phase magnetic fields. These modes can be understood as the hybrid modes of two coupled resonators (apertures): I being the higher energy anti-bonding and II being the lower energy bonding mode. ${ }^{[9]}$ With increasing symmetry breaking the bonding mode II becomes dominated by the longer aperture, see III.

(a) Transmission

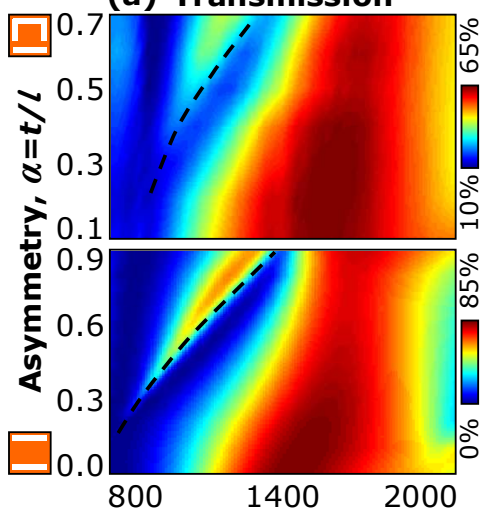

(b) Reflection

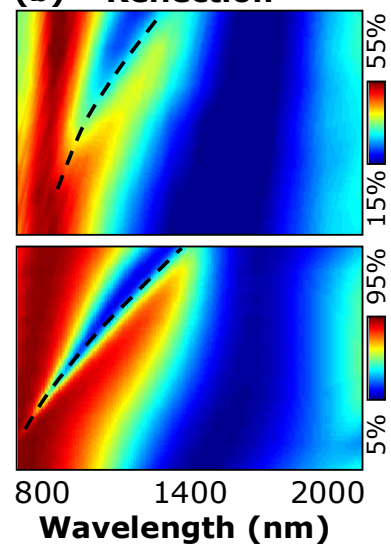

(c) Absorption

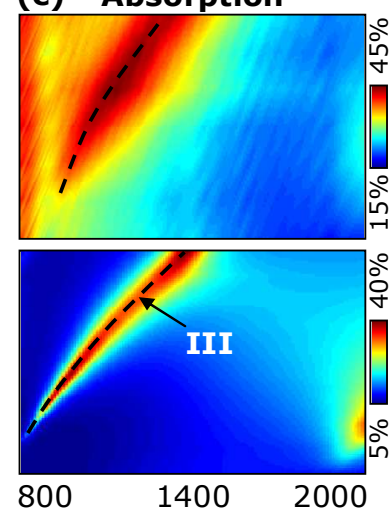

(d) FOM

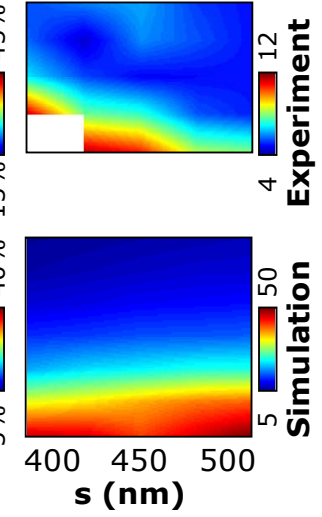

Figure 2. Library A-gap size asymmetry $\alpha=t / l$, where parameters $l=s-200 \mathrm{~nm}$ and $b=0 \mathrm{~nm}$ remain fixed. a) Transmission, b) reflection and c) absorption spectra as a function of symmetry breaking $\alpha$ for unit cell size $s=500 \mathrm{~nm}$. The dashed line corresponds to the absorption maximum. (d) Combinatorial maps of resonance sharpness as a function of unit cell size $s$ versus gap size asymmetry $\alpha$. Here the resonance width is measured as a figure of merit (FOM) defined as the resonance wavelength of the absorption peak divided by its full width half maximum. Note that the experiments (top) cover a slightly smaller range of asymmetries than the simulations (bottom).

As discussed in the introduction, narrow Fano resonances have a wide range of applications. To quantify the width of the bonding resonance we introduce a figure of merit defined as $F O M=\lambda_{0} / \Delta \lambda$, where $\lambda_{0}$ is the resonant wavelength and $\Delta \lambda$ is the full width half maximum of the absorption peak. As illustrated by Figs. $2 \mathrm{~d}$ and $3 \mathrm{~d}$, for both types of symmetry breaking smaller asymmetries lead to narrower Fano resonances, while the resonance width does not strongly depend on the meta-molecule size, which controls the spectral position of the resonance. 
(a) Transmission

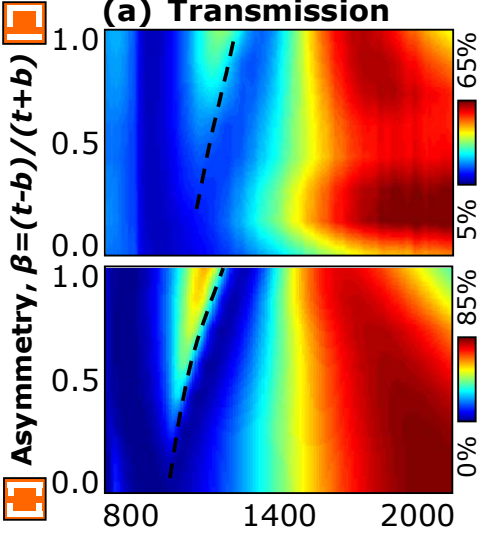

(b) Reflection

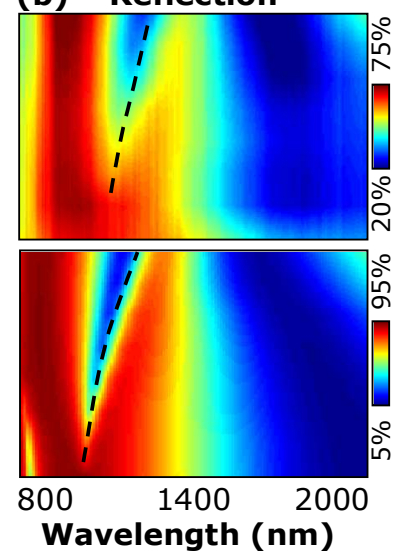

(c)
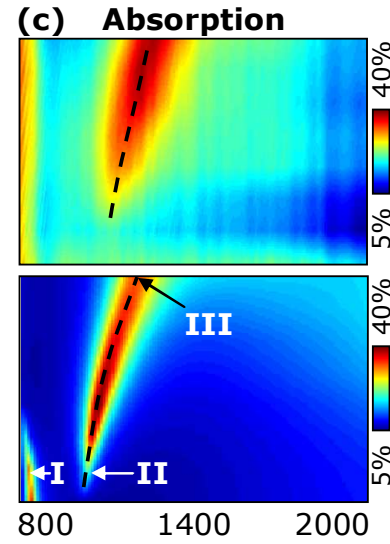

(b) FOM
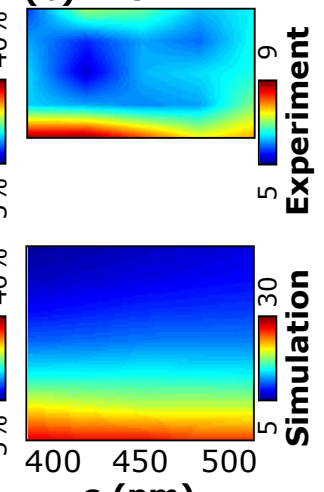

$\mathbf{s}(\mathbf{n m})$

Figure 3. Library $B$ - gap position asymmetry $\beta=(t-b) /(t+b)$, where parameters $l=s-200 \mathrm{~nm}$ and $t+b=0.7 l$ remain fixed. a) Transmission, b) reflection and c) absorption spectra as a function of symmetry breaking $\beta$ for unit cell size $s=500 \mathrm{~nm}$. The dashed line corresponds to the absorption maximum. d) Combinatorial maps of resonance sharpness as a function of unit cell size $s$ versus gap position asymmetry $\beta$. Here the resonance width is measured as a figure of merit (FOM) defined as the resonance wavelength of the absorption peak divided by its full width half maximum.

As illustrated by Figs. 2 and 3, numerical results and experiments show similar qualitative behavior, indicating that simulations do provide useful guidance for the design and understanding of photonic metamaterials. However, quantitative comparison shows substantial differences between experimental and numerical results.

In particular, the spectral position of the measured absorption resonances is red-shifted by about $80 \mathrm{~nm}$ relative to the simulation results. This shift may be explained by uncertainty regarding the real part of the dielectric constant of nanoscale volumes of gold at optical frequencies. Also systematic manufacturing inaccuracies like the inability to fabricate sharp corners at the nanoscale contribute to a spectral shift of resonances, see Fig. 1c-d. In general spectral positions of Fano-type resonances in plasmonic metamaterials are also extremely sensitive to thin cover layers, or layers of oxides or contamination, as has recently been demonstrated by a 3 -fold increase in metamaterial transmission resulting from application of a single layer of graphene. ${ }^{[10]}$

While simulations predict remarkably narrow resonances of up to $F O M=50$ for asymmetries around 0.1 , the experimentally observed resonances are broadened $F O M \leq 12$ and weakened so much, that they cannot be clearly identified for asymmetries $\alpha<0.3$ and $\beta<3 / 7$. Small size variations and manufacturing tolerances in reproducing a perfect square array of meta-molecules, ${ }^{[11]}$ rough gold surfaces as well as finite array sizes ${ }^{[12]}$ were shown to lead to resonance broadening and weakening in real structures, while these factors are not accounted for in simulations of infinite arrays of identical, ideally smooth meta-molecules. Additionally, the width of resonances strongly depends on the imaginary part of the dielectric constant of gold, which may be larger than the commonly used literature values ${ }^{[13]}$ for nanoscale volumes of gold in the optical part of the spectrum due to increased rates of electron scattering. These factors can also explain why the remarkably narrow anti-bonding resonance associated with the symmetric mode of excitation has not been observed in real structures. Furthermore, the above factors can also cause the high background of absorption observed in our real metamaterial samples.

For practical applications a trade-off between narrow Fano resonances (small asymmetry) and strong Fano resonances (large asymmetry) must be considered. The optimal design parameters (i.e. asymmetry) will always depend on the accuracy of the available 
nanostructuring technology and can be found through the high through-put screening method demonstrated here. In our case asymmetries around $\alpha, \beta \approx 0.4-0.5$ are the best choice for most sensor and switching applications, which is in stark contrast to simulations suggesting optimal asymmetries of around 0.1 .

In summary we report a high through-put combinatorial approach to the design and optimization of photonic metamaterials. We show for Fano resonances in split ring aperture arrays, that the optimal metamaterial parameters can be found through parallel synthesis and characterization of material libraries with quasi-continuous variation of design parameters under real manufacturing conditions. Being unable to take the inevitable shortcomings of nanoscale manufacturing into account, simulations can only provide qualitative insights into metamaterial properties and underlying mechanisms. Here we have identified in detail how the asymmetry of split ring apertures controls Fano resonances in photonic metamaterials, which are relevant to various applications ranging from sensing, switches and slow-light devices to the "lasing spaser". ${ }^{[14]}$

\section{Experimental}

Metamaterial libraries: We studied metamaterials consisting of arrays of square split-ring aperture meta-molecules in the field of parameters defined by the overall size of the metamaterial unit cell and the asymmetry of the split (see Fig. 1b). Here $s \times s$ is the overall size of the square unit cell while in all cases the meta-molecule's outer and inner sides $a$ and $l$ were $100 \mathrm{~nm}$ and $200 \mathrm{~nm}$ smaller than the unit cell, respectively. The design slit width $w$ was $50 \mathrm{~nm}$ and parameters $t$ and $b$ define the asymmetry of the split and its size. Two libraries of regular $30 \times 30 \mu \mathrm{m}^{2}$ arrays of split ring apertures in a $30 \mathrm{~nm}$ thick layer of gold were fabricated by electron beam lithography on a glass wafer. In both libraries the unit cell size $s$ was varied from $400 \mathrm{~nm}$ to $500 \mathrm{~nm}$ in steps of $25 \mathrm{~nm}$. In library A parameter $b=0$ was fixed and a gap size asymmetry $\alpha=t / l$ ranging from 0.1 to 0.7 in steps of 0.1 was introduced. In library B the gap size was fixed at $0.3 \mathrm{l}$ and a gap position asymmetry $\beta=(t-b) /(t+b)$ ranging from 0 to 1 in steps of $1 / 7$ was introduced.

Measurements and modeling: The transmission and reflection characteristics of these photonic metamaterials were measured in the spectral range from 800 to $2000 \mathrm{~nm}$ for waves polarized perpendicular to the apertures using a microspectrophotometer. These properties were also simulated with a full three-dimensional Maxwell finite element method solver in the frequency domain using the Drude model for the dielectric constant of gold [13]. 


\section{Acknowledgements}

The authors are grateful to Jun $\mathrm{Yu} \mathrm{Ou}$ for his assistance in imaging our metamaterial samples. Financial support of the Engineering and Physical Sciences Research Council, UK is acknowledged.

\section{References}

[1] X.-D. Xiang, X. Sun, G. Briceño, Y. Lou, K.-A. Wang, H. Chang, W. G. WallaceFreedman, S.-W. Chen, P. G. Schultz, Science 1995, 268, 1738.

[2] V. A. Fedotov, M. Rose, S. L. Prosvirnin, N. Papasimakis, N. I. Zheludev, Phys. Rev. Lett. 2007, 99, 147401.

[3] B. Luk'yanchuk, N. I. Zheludev, S. A. Maier, N. J. Halas, P. Nordlander, H.Giessen, C. Chong, Nature Materials 2010, 9, 707.

[4] C. Rockstuhl, T. Zentgraf, H. Guo, N. Liu, C. Etrich, I. Loa, K. Syassen, J. Kuhl, F. Lederer, H. Giessen, Appl. Phys. B 2006, 84, 219.

[5] K. Aydin, I. M. Pryce, H. A. Atwater, Opt. Express 2010, 18, 13407.

[6] N. Liu, L. Langguth, T. Weiss, J. Kästel, M. Fleischhauer, T. Pfau, H. Giessen, Nat. Materials 2009, 8, 758 .

[7] V. V. Khardikov, E. O. Iarko, S. L. Prosvirnin, J. Opt. 2010, 12, 045102.

[8] N. Papasimakis, N. I. Zheludev, Opt. \& Photonics News 2009, 20, 22.

[9] H. Liu, T. Li, S.-M. Wang, S.-N. Zhu, Front. Phys. China 2010, 5, 277.

[10] N. Papasimakis, Z. Luo, Z. X. Shen, F. D. Angelis, E. D. Fabrizio, A. E. Nikolaenko, N. I. Zheludev, Opt. Exp. 2010, 18, 8353.

[11] N. Papasimakis, V. A. Fedotov, Y. H. Fu, D. P. Tsai, N. I. Zheludev, Phys. Rev. B 2009, 80, 041102(R).

[12] V. A. Fedotov, N. Papasimakis, E. Plum, A. Bitzer, M. Walther, P. Kuo, D. P. Tsai, N. I. Zheludev, Phys. Rev. Lett. 2010, 104, 223901.

[13] P. B. Johnson, R. W. Christy, Phys. Rev. B 1972, 6, 4370.

[14] N. I. Zheludev, S. L. Prosvirnin, N. Papasimakis, V. A. Fedotov, Nat. Photonics 2008, 2,351 . 